\title{
Steven A. Koehler, Peggy A. Brown: Forensic epidemiology
}

\author{
CRC Press, Taylor and Francis Group, 287 pp, hardback, ISBN 978-1-4200-6327-1
}

\author{
Sharon M. Derrick
}

Accepted: 12 January 2010/Published online: 27 February 2010

(C) Springer Science+Business Media, LLC 2010

Forensic Epidemiology is an excellent introductory and reference text regarding this growing subfield of epidemiology. The authors provide a clear and accurate description of medical examiner/coroner (ME/C) office procedures as they relate to the categories and quality of mortality data that are utilized in forensic epidemiological practice and research. The volume is well-organized, well-researched and highly readable.

The book begins with an introduction to the forensic death investigation system in order to show that the information routinely gathered by $\mathrm{ME} / \mathrm{C}$ offices is related to that collected during an epidemiological investigation. Therefore, these data are highly valuable to epidemiological research. The chapters that follow build a foundation on basic epidemiological methodology, discuss the potential role of the forensic epidemiologist in the ME/C office, describe in detail the process by which ME/C classify cause and manner of death and what those classifications mean to epidemiologists, and provide examples of epidemiological analysis using forensic mortality data.

One comprehensive example of epidemiological investigation using mortality data is provided by the discussion of fall fatalities in the chapter on accidental deaths (Chapter 7). Accidental falls are a major source of preventable injury and death across the lifespan in the United States. The take-away point from this discussion is that utilization of death certificate data alone does not provide the epidemiologist with the rich information regarding the circumstances of the fall that is provided through $\mathrm{ME} / \mathrm{C}$

S. M. Derrick ( $₫)$

1885 Old Spanish Trail, Houston, TX 77054, USA

e-mail: sharon.derrick@meo.hctx.net

URL: http://www.hctx.net/me investigative procedure. The circumstances that result in a fall inform fall prevention efforts globally.

Although I would recommend this book to epidemiologists, students and others interested in the application of epidemiology to forensics, there are a few minor flaws in the volume that should be mentioned. One is the use of "an ME/C office" case information in certain charts (see Table 6.2, page 77 for an example) without a clear label noting the origin of the data. These numbers are without context regarding the size of the $\mathrm{ME} / \mathrm{C}$ office or demographic and geographic factors.

Possibly in an attempt to simplify forensic terms or descriptions of forensic procedures, some definitions are too generalized so as to be either incorrect or inapplicable across the ME/C office spectrum. For example, despite the spot-on use of the fall example described above, the authors state on page 138 that "A fall is defined as blunt force trauma (BFT) inflicted when an individual tumbles..." and further, "The result of any fall is blunt force trauma." Although fatal falls routinely involve BFT, the cause of death in a fall can also be another type of injury, for example, sharp force trauma from impalement on a sharp object with a mechanism of exsanguination. Another example of oversimplification is the inclusion of heatrelated deaths in the Chapter 6 discussion of natural deaths. Many ME/C classify these deaths as accidental in manner. This inconsistency deserves a caveat in the text to address any possible confusion the classifications may cause researchers.

In sum, Koehler and Brown's Forensic Epidemiology is a much-needed addition to the epidemiological and forensic literature. The well-written and researched volume provides a useful textbook option for college students and a handy office reference for professionals. 